Les sources de l'Histoire des Mines : Nouveaux outils, Nouvelles approches

\title{
A. P. Ledger et Roger Smith, Benjamin Vulliamy and the Derby porcelain manufactory, 1784-1795
}

Derby, Derby museum \& art gallery, 2007, 142 pages.

\section{Liliane Pérez}

\section{(2) OpenEdition \\ Journals}

Édition électronique

URL : http://journals.openedition.org/dht/733

DOI : $10.4000 /$ dht.733

ISSN : $1775-4194$

Éditeur :

Centre d'histoire des techniques et de l'environnement du Cnam (CDHTE-Cnam), Société des élèves du CDHTE-Cnam

\section{Édition imprimée}

Date de publication : 1 décembre 2008

Pagination : 226-229

ISBN : 978-2-95-30779-2-6

ISSN : 0417-8726

Référence électronique

Liliane Pérez, « A. P. Ledger et Roger Smith, Benjamin Vulliamy and the Derby porcelain manufactory, 1784-1795 », Documents pour I'histoire des techniques [En ligne], $16 \mid 2^{\mathrm{e}}$ semestre 2008, mis en ligne le 05 octobre 2010, consulté le 24 septembre 2020. URL : http://journals.openedition.org/dht/733 ; DOI : https://doi.org/10.4000/dht.733

Ce document a été généré automatiquement le 24 septembre 2020

(C) Tous droits réservés 


\section{A. P. Ledger et Roger Smith, Benjamin Vulliamy and the Derby porcelain manufactory, 1784-1795}

Derby, Derby museum \& art gallery, 2007, 142 pages.

\section{Liliane Pérez}

\section{RÉFÉRENCE}

A. P. Ledger et Roger Smith, Benjamin Vulliamy and the Derby porcelain manufactory, 1784-1795, Derby, Derby museum \& art gallery, 2007, 142 pages.

1 En Angleterre, au XVIII ${ }^{\mathrm{e}}$ siècle, l'essor consumériste et le processus de différenciation de produits qui le sous-tend sont étroitement liés au dynamisme d'entreprises sectorielles qui étoffent les services proposés aux clientèles et développent la production de gammes d'articles diversifiés et composites, fondés sur des assemblages de pièces et de matières, notamment dans les domaines de l'horlogerie et du toyware (secteur allant des accessoires vestimentaires aux décorations intérieures et aux ustensiles de cuisine, en passant par l'instrumentation). Des firmes complexes, appelées "comprehensive manufacturing firms" par l'historienne du meuble Pat Kirkham, se mettent en place grâce à l'intensification des marchés de production, donc des échanges de semi-produits, d'ébauches, de pièces et d'attaches, mais aussi d'informations (dessins, modèles, patrons). Au cœur de cette économie de la variété, se tient le renforcement des complémentarités entre métiers, entre entreprises, entre secteurs, en somme une dynamique de coordination, fondée sur de multiples formes d'association, à différentes échelles : dans la capitale (le fait est bien connu pour le district de Westminster), entre les régions, ainsi Birmingham et la zone des Potteries comme le suggèrent les échanges, bien connus maintenant, entre Matthew Boulton et Josiah Wedgwood, étudiés par Maxine Berg. 
2 Le catalogue édité par A. P. Ledger et Roger Smith permet de cerner un autre grand réseau d'entrepreneurs anglais, entre Benjamin Vulliamy (1747-1811), horloger, orfèvre et décorateur de luxe installé avec son fils à Londres, sur Pall Mall, et William Duesbury II (1763-1796), manufacturier de porcelaine du Derbyshire ${ }^{1}$. Si les liens des Vulliamy avec Wedgwood et les manufacturiers du Derbyshire sont maintenant bien connus, ainsi que leur insertion dans l'économie londonienne du meuble, de l'argenterie et des bronzes, les publications à ce sujet restaient limitées à des articles pionniers parus dans des revues spécialisées. Le catalogue de A. P. Ledger et Roger Smith offre pour la première fois une synthèse facile d'accès sur les échanges entre ces entrepreneurs, assortie de l'édition commentée d'archives privées de la firme Duesbury de Derby entre 1784 et 1795, extraites des registres comptables de l'entreprise, day books, et de la correspondance de Benjamin Vulliamy, principal client de la firme, avec William Duesbury (16 lettres, toutes reproduites), ou bien de Joseph Lygo, directeur de l'entrepôt londonien de Duesbury et actif intermédiaire de Vulliamy (plus de 100 lettres concernant les commandes de l'horloger). Ces archives, contenues dans les Duesbury papers (Derby local studies library), sont assorties de photographies des horloges de Vulliamy ornés de porcelaines de Derby, conservées dans plusieurs collections et citées dans ces documents. Le catalogue permet ainsi d'associer les écrits et les objets, et de comprendre quels marchés et quelles médiations, révélés par les textes, ont sous-tendu la production d'articles composites et raffinés dont la production nécessite la coordination de circuits complexes d'approvisionnement et d'information. Le fait de pouvoir repérer avec précision les modèles mentionnés dans les archives et d'avoir réuni leurs clichés grâce à un travail d'identification dans différents dépôts et collections, constitue l'originalité de ce recueil ${ }^{2}$.

3 Après un chapitre de synthèse, l'ouvrage présente ces différentes archives, écrits et artefacts, année par année, de 1784 à 1795. Comme le soulignent les auteurs, les lettres de Vulliamy (et de Lygo) confirment le rôle de gestionnaire rigoureux de l'horloger décorateur dans le système d'échanges mis sur pied avec Duesbury, ainsi qu'avec les sculpteurs de la capitale, chargés de réaliser les modèles imaginés, dessinés, par Vulliamy, notamment trois grands artistes, issus des écoles de la Royal Academy, Henry Webber (devenu directeur du département d'ornement de Wedgwood), John Deare et Charles Peart. Si Vulliamy ne tient pas d'atelier ni de manufacture à Londres, si son affaire repose sur l'extension de la sous-traitance, fondement de l'élargissement des gammes d'objets dans les métiers du luxe, ses compétences marchandes se conjuguent à celles d'organisation de la production : Vulliamy impose au manufacturier de Derby ses standards de qualité ; il conçoit lui-même les dessins des ornements, et les modèles réalisés par les sculpteurs londoniens, acheminés à Derby, restent toujours sa propriété ; enfin, il supervise toute relation (notamment la correspondance) entre ces modeleurs et William Duesbury. Ce contrôle entrepreneurial s'exerce donc à distance, hors de toute structure de production concentrée et repose sur l'écrit prescriptif, les dessins et les modèles (devant être imités de la manière la plus exacte). Vulliamy intervient de plus, dans le recrutement des artistes et des ouvriers de talent à Derby. Pour autant, la coordination n'est pas aisée comme le rappellent les auteurs ; Duesbury réplique ainsi dans une lettre à Vulliamy : «ce n'est pas dans la nature de la porcelaine d'être commandée " ( it is not in the power of porcelain to be commended»), tant la fabrication des biscuits (sans émaillage ni couleurs, donc sans possibilité de masquer les défauts), en pâte dure (avec kaolin) et en grand format (20 à 30 centimètres), à l'imitation de Sèvres, pose de problèmes dans l'Angleterre des années 1780. Mais 
l'essentiel des échanges entre Vulliamy et Duesbury porte avant tout sur la combinaison d'éléments, notamment l'ajustage des pièces de porcelaine de Derby et de celles de marbre ou d'ormoulu issues de fournisseurs londoniens (s'y ajoute, à Derby, le travail délicat d'assemblage des pièces de porcelaine composant ces œuvres complexes, avant la mise au feu, une tâche dévolue à des ouvriers spécialisés, les «repairers »). Si l'entrepreneur prévoit des éléments amovibles - ainsi, les mains et les bras des figures, conservés au Spode museum, à Stoke-on-Trent, dont le catalogue publie des clichés facilitant les problèmes d'enfournement des groupes, la précision et la conformité des pièces aux instructions restent des exigences difficiles à mettre en œuvre, surtout à l'échelle d'un vaste réseau. Néanmoins, les demandes de Vulliamy initient une dynamique d'amélioration et de perfectionnement à Derby, tant les grandes figures de porcelaine ainsi que le respect de normes et de spécifications (qualité de la pâte, dimensionnement des pièces) représentent de défis techniques. S'y ajoute la tonalité néo-classique voulue par Vulliamy (à l'image de l'horlogerie française et des productions de Sèvres, alors que les modèles prisés à Derby sont rococo). L'horloger londonien, puisant parfois dans les stocks de Duesbury, n'hésite pas remplacer des éléments par d'autres plus à son goût. Au fil de ces échanges, s'exprime une culture de la composition, de la substitution et de la copie (également à partir des recueils d'antiques de la bibliothèque de Vulliamy), clés de voûte de l'économie du produit et des capacités inventives des Lumières.

4 Les photographies d'horloges, issues de diverses collections, privées et publiques (Victoria \& Albert museum, Spode museum, Sotheby's, Mallett, etc.) sont particulièrement bien conçues. En effet, plus que le bel objet, c'est le geste et la technique qui ont concouru à sa fabrication qui sont mis en valeur. Une attention spéciale est portée aux assemblages, y compris par le recours à la radiographie d'articles qui permet de déceler les broches et les joints utilisés par l'entrepreneur pour fixer ses compositions. D'autres clichés révèlent les agencements imaginés par Vulliamy, ainsi les variations de figures d'un groupe à un autre (tel support, tel personnage sont remplacés); les légendes guident utilement le lecteur dans la compréhension de cette combinatoire. Enfin, les techniques d'ajustage sont soulignées grâce à des photographies en gros plan des zones de contact entre ormoulu et porcelaine, par exemple ; tel soubassement de colonne métallique a été retaillé sur trois millimètres pour faire une place au pied d'une figure. L'encastrement des pièces d'horlogerie dans les décors de porcelaine est aussi mis à jour grâce aux clichés des mécanismes.

5 La publication de la correspondance (essentiellement composée de lettres de Vulliamy) est aussi présentée avec intelligence. L'origine de chaque document apparaît clairement au bas du texte et les éditeurs ont pris soin de mentionner les transcriptions réalisées par Alan Green (déposées à la bibliothèque de Derby). Lorsque des archives ont disparu, les notes d'Alan Green et celles de Thimothy Clifford (1990) ont été utilisées, autorisant ainsi la restitution d'une continuité des échanges épistolaires entre 1784 et 1795, une période peu couverte par les papiers de la firme Vulliamy conservés notamment à Kew (The National Archives), qui portent surtout sur le début du XIX ${ }^{\mathrm{e}}$ siècle. Il s'agit d'une véritable édition critique, chaque lettre étant assortie de plusieurs notes explicatives, parfois denses, pour commenter telle technique, tel ornement. S'y ajoutent les notes de renvoi entre les pièces citées dans les archives et identifiées dans des collections (photographies à l'appui). En annexe, sont récapitulées les ventes de figures listées au fil de la correspondance, suivies de la mention des achats de Vulliamy 
repérés dans les day books de Duesbury, puis de plusieurs tableaux (valeur annuelle des achats d'ornements par Vulliamy, estimation de ses paiements, comparaison des livraisons faites à Vulliamy et des figures effectivement utilisées pour ses horloges). La deuxième annexe concerne les vases uniquement, et la troisième offre une synthèse sur les productions de Derby, notamment sur les techniques de fabrication des figures. Les éditeurs estiment que malgré les prix élevés payés par Vulliamy, le coût de production des grandes figures en biscuit rend ce marché peu profitable pour Duesbury. Le volume se clôt par un index biographique détaillé, véritable mine d'informations, et sur un index des objets tout aussi bien renseigné (y compris pour les pièces détachées). Cette édition commentée est un modèle du genre.

Dès la première lettre, de Benjamin Vulliamy à William Duesbury (31 mai 1784), apparaissent toutes les qualités de cette publication. Le document, disparu dans les Duesbury papers, est restitué à partir des notes de T. Clifford et A. Green. Comme l'horloger prescrit des indications précises au manufacturier de Derby pour l'huilage des pièces composant un ornement, les éditeurs mentionnent que cette œuvre a pu être identifiée comme étant Angel et Urania, actuellement conservée par la société de vente Mallett, et dont la photographie illustre la couverture du volume. Vulliamy prescrit que l'ange doit porter des ailes amovibles, pour lesquelles Duesbury est chargé de soigner les joints ; les éditeurs confirment en bas de page que ce personnage fut bien livré avec et sans ailes. Sept notes explicatives permettent de comprendre plusieurs allusions de Vulliamy, ainsi celle au service de la manufacture de Sèvres, admiré et soupesé par l'entrepreneur lors d'un souper chez le prince de Galles, à Carlton House. Les éditeurs apportent des indications sur cette festivité et sur le service de Sèvres, présent diplomatique adressé par le comte d'Angiviller; ils complètent l'information par des annonces parues dans la presse à l'occasion de cette cérémonie; ils offrent enfin un état comparé des fabrications de porcelaine entre France et Angleterre, la première surpassant largement la seconde pour la qualité du biscuit, spécialité de Sèvres, grâce à la maîtrise des pâtes dures, sujettes à moins de variations à la cuisson que les pâtes tendres, et offrant aussi une couleur plus blanche (à l'imitation du marbre). Vulliamy exprime sans détour son éloge de la porcelaine française et son désir de l'imiter, dans le droit fil de la politique économique anglaise des substituts d'importation, prônée par exemple par la Society of arts de Londres, comme l'a montré Maxine Berg. L'entrepreneur compare ainsi les décors de Derby, aussi exposés lors de la fête du prince de Galles, et les pièces de Sèvres, pour inviter Duesbury à prendre exemple sur les productions françaises. Très utilement, les éditeurs du catalogue réussissent à identifier ce service de Derby, à partir des factures contenues dans les Royal Archives (Windsor Castle), et en fournissent le détail complet. La culture de la copie, principe fondamental chez Vulliamy, transparaît également des exigences de l'entrepreneur pour que les figures faites à Derby soient les plus fidèles possible aux modèles fournis par le sculpteur londonien John Deare.

7 L'insistance sur les techniques d'ajustage, notamment les joints et leur huilage, la conjonction entre les arts et les techniques au nom du dessein - «design» - de l'objet, la comparaison entre les qualités des productions de Sèvres et de Derby, l'exigence de copie des modèles londoniens : autant de thèmes qui reviennent, avec des variantes, au fil des autres lettres, et qui inscrivent l'économie du produit, portée par les métiers du luxe, dans une compréhension synthétique, combinatoire et analogique des objets et des processus de fabrication, sous l'égide d'un entrepreneur marchand, homme de réseau, gestionnaire et directeur effectif de la fabrication. Notons que les auteurs/ 
éditeurs sont des historiens de l'art dont les travaux (notamment les nombreux articles de Roger Smith) ont joué un rôle de premier plan dans l'analyse de la culture technique des entrepreneurs marchands anglais au XVIII ${ }^{e}$ siècle, promoteurs de savoirs d'organisation qui étaient traditionnellement associés dans l'historiographie à l'essor du machinisme et des productions en série. Au contraire, c'est l'économie de la variété, de l'ornement et de la performance qui promeut des compétences croissantes d'assemblage, de coordination (des pièces, des matériaux, des gestes), de transposition et de substitution - en somme une intelligence des rapports, rameau de la pensée complexe qui participe, sur le long terme, de l'affirmation des sciences de l'artificiel, du projet et de l'ingénierie ${ }^{3}$.

\section{NOTES}

1. Les études pionnières ont été menées par Timothy Clifford : "Vulliamy clocks \& British sculpture ", Apollo, vol. 132, 1990, pp. 226-237 et "New evidence concerning Vulliamy clocks and Duesbury porcelain", Journal of the Derby porcelain international Society, 1991, pp. 35-52.

2. Il forme le troisième d'une série, les Derby manufactory archive research reports, dont les deux premiers offraient une étude des relations commerciales de la manufacture de Derby sous William Duesbury II.

3. Herbert A. Simon, Les sciences de l'artificiel (1996), Paris, Folio/Essais, 2001.

\section{AUTEURS}

LILIANE PÉREZ

CDHTE-Cnam 